Revue internationale P.M.E.

Économie et gestion de la petite et moyenne entreprise

Revure

internationale

PME

\title{
Micromentalités et logiques d'action des entrepreneurs dirigeants de petites entreprises
}

\section{Malek Bouhaouala}

Volume 20, numéro 2, 2007

URI : https://id.erudit.org/iderudit/1008519ar

DOI : https://doi.org/10.7202/1008519ar

Aller au sommaire du numéro

Éditeur(s)

Presses de l’Université du Québec

ISSN

0776-5436 (imprimé)

1918-9699 (numérique)

Découvrir la revue

Citer cette note

Bouhaouala, M. (2007). Micromentalités et logiques d'action des entrepreneurs dirigeants de petites entreprises. Revue internationale P.M.E., 20(2), 123-149. https://doi.org/10.7202/1008519ar
Résumé de l'article

Cet article présente, dans une approche empirique et inductive, l'application du concept de micromentalités des entrepreneurs dirigeants à la compréhension de l'univers des petites et très petites entreprises (PE-TPE). Dans un premier temps, cela a consisté à reconstruire les micromentalités à partir des objectifs, valeurs et conceptions des entrepreneurs et de relever les caractéristiques les plus discriminantes dans le fonctionnement des PE-TPE (formes organisationnelles, stratégies et actions commerciales). Dans un second temps, il s'est agi de confronter les deux niveaux d'analyse subjectif (micromentalités) et objectif (caractéristiques des PE-TPE) pour vérifier l'efficacité et la force explicative de l'approche par les micromentalités des entrepreneurs. Il en est ressorti une typologie multicritères composée de quatre classes d'entrepreneurs dirigeants et de PE-TPE. Chaque type est distingué par une micromentalité et un mode de gestion spécifique se caractérisant par une logique d'action, une forme d'organisation, une stratégie et une démarche marketing propres. Les résultats de la recherche exposés partiellement et d'une manière synthétique ici montrent, d'une part, l'importance de la prise en compte des facteurs non économiques inhérents aux entrepreneurs et, d'autre part, l'incapacité des seuls facteurs objectifs et abstraits à fournir une analyse nuancée et réaliste de la gestion de la petite entreprise.
Ce document est protégé par la loi sur le droit d'auteur. L'utilisation des services d'Érudit (y compris la reproduction) est assujettie à sa politique d'utilisation que vous pouvez consulter en ligne.

https://apropos.erudit.org/fr/usagers/politique-dutilisation/ 


\title{
Micromentalités et logiques d'action des entrepreneurs dirigeants de petites entreprises
}

Malek BOUHAOUALA

SENS, Université Joseph-Fourier, Grenoble I

\section{MOTS CLÉS}

Micromentalités - Logiques d'action - Typologie multicritères

Approche inductive - PE-TPE

\begin{abstract}
L'AUTEUR
MaLeK Bouhaouala détient un Ph.D. et est maître de conférences à l'Université Joseph-Fourier Grenoble I. Il enseigne et mène des recherches sur le domaine de l'entrepreneuriat dans les secteurs des loisirs et du tourisme sportif. Il a publié des articles sur les logiques d'action des entrepreneurs-dirigeants, les stratégies des PE-TPE, la régulation des marchés locaux du tourisme sportif et l'entrepreneuriat en loisirs et tourisme équestre. Il conduit actuellement des travaux sur le développement local par le sport et sur le processus de création d'entreprise dans le secteur socioéconomique des loisirs sportifs. Adresse: SENS, Université JosephFourier Grenoble I, UFR APS, B.P. 53, 38041 Grenoble Cédex 9, France. Tél. : 334766350 90. Courriel :<malek.bouhaouala@ujf-grenoble.fr>. Site Web :<http://www-sens.ujf-grenoble. fr/annuaire/bouhaouala.html>.
\end{abstract}

\section{RÉSUMÉ}

Cet article présente, dans une approche empirique et inductive, l'application du concept de micromentalités des entrepreneurs dirigeants à la compréhension de l'univers des petites et très petites entreprises (PE-TPE). Dans un premier temps, cela a consisté à reconstruire les micromentalités à partir des objectifs, valeurs et conceptions des entrepreneurs et de relever les caractéristiques les plus discriminantes dans le fonctionnement des PE-TPE (formes organisationnelles, stratégies et actions commerciales). Dans un second temps, il s'est agi de confronter les deux niveaux d'analyse subjectif (micromentalités) et objectif (caractéristiques des PE-TPE) pour vérifier l'efficacité et la force explicative de l'approche par les micromentalités des entrepreneurs. II en est ressorti une typologie multicritères composée de quatre classes d'entrepreneurs dirigeants et de PE-TPE. Chaque type est distingué par une micromentalité et un mode de gestion spécifique se 
caractérisant par une logique d'action, une forme d'organisation, une stratégie et une démarche marketing propres. Les résultats de la recherche exposés partiellement et d'une manière synthétique ici montrent, d'une part, l'importance de la prise en compte des facteurs non économiques inhérents aux entrepreneurs et, d'autre part, l'incapacité des seuls facteurs objectifs et abstraits à fournir une analyse nuancée et réaliste de la gestion de la petite entreprise.

\section{ABSTRACT}

This paper presents, through an empirical and inductive approach, the application of the concept of micro-mentalities of the entrepeneurs to make understable the reality of small firms and very small firmes' (SF-VSF) universe. Initially, that consisted in rebuilding micro-mentalities based on the objectives, values and conceptions of the entrepreneurs and to point out the most discriminating characteristics in the functioning of the SF-VSF (organisational forms, commercial strategies and actions). In the second time, we have confronted the two levels of analysis subjective (micro-mentalities) and objective (characteristics of the SF-VSF) to check the effectiveness and the explanatory force of the approach by micro-mentalities. It is come out from it, a multicriterion typology made up of four classes of entrepreneurs and SF-VSF. Each type is distinguished by a micro-mentality and a form of management and being characterized by a specific logic from action, form of organization, strategy and marketing action. The results of this research, exposed partially here, show on one hand, the importance of the taking into account of the not-economic factors inherent to the entrepreneur, and on the other hand, the weakness of the only objective and abstract factors to provide a finely-shaded and realistic analysis of SF-VSF forms of management.

\section{RESUMEN}

Este artículo presenta un enfoque empírico e inductivo, la aplicación del concepto micro-mentalidades de los empresarios dirigentes a la comprensión del universo de las pequeñas firmas. Inicialmente consistió en reconstruir las micro-mentalidades a partir de los objetivos, valores y concepciones de los empresarios y de destacar las características que más discriminaban en el funcionamiento de las pequeñas firmas (formas organizativas, estrategias y acciones comerciales). Posteriormente, desde se trata de enfrentar los dos niveles de análisis subjetivo (micro-mentalidades) y objetivo (características de los firmas) para comprobar la eficacia y la fuerza explicativa del enfoque por las micro-mentalidades de los empresarios. Como resultado, una tipología multicriterio compuesta de cuatro clases de empresarios dirigentes y pequeñas firmas. Cada tipo, se distingue por una micro-mentalidad y un método de gestión específico que se caracteriza por una lógica de acción, una forma de organización, una estrategia y un acción de maketing. Los resultados de la investigación expuestos parcialmente y de una manera sintética aquí, muestran por una parte, la importancia de la consideración de los factores no económicos inherentes a los empresarios, y de otro, la incapacidad de los factores objetivos porsi solos, y abstractos que deben proporcionarse para un análisis, moderado y realista de la gestión de la pequeña firma. 


\section{ZUSAMMENFASSUNG}

Dieser Artikel stellt in einem empirischen und induktiven Ansatz die Anwendung des Konzeptes von Mikro-Mentalitäten bei leitenden Unternehmern vor, um das Universum kleiner und sehr kleiner Unternehmen (PE-TPE) zu verstehen. Zunächst galt es, die Mikro-Mentalitäten anhand der Zielsetzungen, Werte und Konzeptionen der Unternehmer aufzubauen, und die Eigenschaften hervorzuheben, die für das Funktionieren der kleinen und sehr kleinen Unternehmen zentral sind (organisatorische Formen, Strategien und Handelsaktionen). In einem zweiten Schritt wurden die zwei Analyseniveaus subjektiv (Mikro-Mentalitäten) und objektiv (Eigenschaften der Unternehmen) konfrontiert, um die Wirksamkeit des Ansatzes zu prüfen. Daraus ist eine multiple Typologie hervorgegangen, die sich aus vier Klassen leitender Unternehmer und PE-TPE zusammenstellt. Jeder Typ wird gekennzeichnet durch eine eigene Mikro-Mentalität und einem spezifischen Führungsstil, der sich durch die Aktionslogik, der Organisationsart, der Strategie und dem Marketingvorgehen charakterisiert. Die angeführten Forschungsergebnisse zeigen einerseits die Bedeutung der nicht-wirtschaftlichen Faktoren und Charaktereigenschaften, andererseits die Unzulänglichkeit der objektiven und abstrakten Faktoren, eine nuancierte und realistische Analyse des Managements für kleine und sehr kleine Unternehmen zu liefern.

\section{Introduction}

Au regard de la littérature issue de la sociologie des professions (Bauer, 1993; Gresle, 1981;Zarca,1986), on ne peut traiter la question des comportements économiques des entrepreneurs dirigeants de petites et très petites entreprises (PE-TPE) en faisant uniquement référence à la théorie économique et à des explications en termes économiques. L'idée selon laquelle la subjectivité des entrepreneurs dirigeants permet de comprendre les modes de gestion des petites entreprises et leurs déviances et/ou contradictions par rapport à la rationalité économique n'est pas nouvelle.

Les objectifs, les aspirations et le profil des dirigeants (Julien et Marchesnay, 1988, 1996), l'indépendance (Gresle, 1981), la mentalité artisanale traditionnelle (Zarca, 1986), la transmission de l'entreprise (patrimoine familial) aux générations futures (Bauer, 1993), la passion (Bouhaouala et Chifflet, 2001) constituent des alternatives explicatives aux seuls paramètres du marché dans l'analyse des comportements économiques et professionnels des entrepreneurs dirigeants.

Ces travaux ont montré que le raisonnement des entrepreneurs dirigeants n'est pas toujours en accord avec les modèles de raisonnement de la théorie économique. En effet, les projets personnels des entrepreneurs dirigeants influencent la gestion et le développement de la petite entreprise (Bouhaouala, 1999; Collins et Randolph, 1991; Gresle, 1981; Lahlou, Maffre et Moati, 1991; Moati, 1993; Zarca, 1986). Leurs comportements peuvent 
parfois paraître anti-économiques (Bauer, 1993; Gresle, 1981;Zarca, 1986), inefficaces du point de vue de la concurrence sectorielle (Porter, 1982) ou désintéressés (Herscovici, 1994). En d'autres termes, bien qu'ils produisent des effets économiques et commerciaux sur les marchés, leurs comportements ne semblent pas être souvent conformes au canon de la rationalité économique.

Ce texte présente une approche explicative fondée sur le concept de micromentalités ${ }^{1}$ des entrepreneurs dirigeants ayant servi à comprendre l'univers des PE-TPE du secteur du tourisme sportif (Bouhaouala, 1999). En effet, les logiques d'action et les comportements qui en découlent sont à considérer ici du point de vue d'une détermination relative, dans le sens où leurs visions subjectives du monde économique et professionnel ne sont pas isolées de l'environnement social et économique de l'entreprise. La conceptualisation des micromentalités permet de les replacer dans des ensembles de significations relatives à des groupes restreints d'entrepreneurs dirigeants partageant la même micromentalité. L'explication des modes de gestion des PE-TPE ne serait plus monocausale, c'est-à-dire fondée uniquement sur les paramètres du marché et cohérente avec une rationalité économique abstraite. L'explication est fondée sur une causalité multiple permettant de rendre compte de la complexité de la réalité des PE-TPE et des logiques d'action pratiques et contradictoires par rapport au modèle de l'homo æeconomicus.

\section{De l'existence à l'efficacité des micromentalités des entrepreneurs}

Des auteurs classiques (Weber, Simiand, Schumpeter, etc.) ont, de leur côté, utilisé la notion de mentalité sous des formes variées et sans la définir d'une manière univoque. Cependant, l'idée de l'influence des mentalités sur les comportements de groupes sociaux importants n'est plus à démontrer (Gislain et Steiner, 1995).

Schumpeter (1911), dans sa Théorie de l'évolution économique, à partir de la définition de l'entrepreneur, a mis en évidence une certaine mentalité qui caractérise ce dernier. L'entrepreneur de Schumpeter se distingue alors de l'agent économique rationnel. Il se caractérise plutôt par sa relative liberté à l'égard des paramètres du marché, par sa capacité importante de prise de risque et d'innovation, par sa créativité et sa ténacité. En l'absence de ces

1. La subjectivité associée à la conception du monde et des objets, à la vision des choses ou à la manière de penser la réalité sociale, propre à des groupes particuliers, est conceptualisée ici sous le terme de micromentalités. Mais l'utilisation de ce concept nécessite de le différencier d'autres notions proches et plus souvent utilisées - la culture, les mentalités, les représentations sociales - pour mieux souligner sa spécificité théorique. 
caractéristiques, il n'entreprendrait que des actions qui tendent vers le risque zéro. L'entrepreneur, à travers sa mentalité, présente un comportement dynamique (non conforme à celui de l'homo æeconomicus) et devient ainsi l'un des éléments clés de l'évolution du système économique.

Max Weber, dans son ouvrage L'éthique protestante et l'esprit du capitalisme, parle de mentalités pour montrer le rôle de l'éthique calviniste dans l'évolution du capitalisme occidental. Sa thèse est que l'esprit calviniste forme une mentalité économique particulière qu'il désigne par l'ethos d'une forme d'économie (Weber, 1964, p. 21) qui a favorisé la naissance du capitalisme occidental au cours des XVI ${ }^{\mathrm{e}}$ et XVII ${ }^{\mathrm{e}}$ siècles (Weber, 1964). Ce dernier n'exclut ni les facteurs économiques ni le rationalisme économique, mais il les considère plutôt comme des conditions importantes pour le développement économique et non comme des conditions essentielles. Il apparaît donc que ce développement dépendrait davantage de «la faculté et des dispositions qu'a l'homme d'adopter certains types de conduites rationnelles pratiques » (Weber, 1964, p. 21).

Dans la même lignée, le travail de François Simiand $(1932,1934)$ met en avant le rôle des conceptions du juste dans l'orientation des comportements des acteurs sociaux sur les marchés. Pour Simiand, ces conceptions orientent en partie les comportements économiques et expliquent par conséquent la formation et l'acceptation des prix de référence et du salaire nominal. Les conceptions du juste et des transactions mêmes participeraient alors à la formation des mentalités propres à des groupes sociaux. Les luttes pour les salaires sont ainsi orientées par le sentiment subjectif des agents que leur besoin ressenti ou leur peine dépensée est ou n'est pas justement estimé et rétribué (Gislain et Steiner, 1995). En d'autres termes, le sentiment de ce qui est juste serait propre à chaque groupe d'acteurs et la diversité des actions économiques serait relative aux mentalités et au sens donné par chaque groupe à la notion de juste.

L'apport du concept de mentalités économiques se révèle donc à deux niveaux. Premièrement, il permet d'expliquer l'évolution des systèmes sociaux et économiques. En effet, les mentalités ont joué un rôle déterminant dans la formation du système capitaliste occidental (Weber, 1964). Deuxièmement, il permet de comprendre ce que le fonctionnement du marché ou de l'entreprise doit aux subjectivités des acteurs ou de groupes d'acteurs restreints (Simiand, 1932). Quant à l'étude de Schumpeter (1911), on peut dire qu'elle peut concerner les deux niveaux d'analyses. En effet, ce dernier a mis en relation l'évolution du capitalisme avec les mentalités (déviantes) des entrepreneurs. L'usage du concept de mentalités permet donc de montrer l'articulation de facteurs économiques et extra-économiques dans l'orientation des comportements économiques. 


\section{Définition du concept de micromentalités}

Les micromentalités sont définies ici comme un concept synthétique qui regroupe un ensemble non systématiquement cohérent de valeurs, de conceptions (de l'économie, de l'entreprise, du profit, etc.), de motifs et de buts qui contribuent à créer une vision subjective du monde chez les entrepreneurs dirigeants de PE-TPE. Les micromentalités sont alors susceptibles d'être spécifiques et partagées au sein de petits groupes d'entrepreneurs dirigeants. Ainsi, ces derniers, parce qu'ils ont les mêmes conceptions et valeurs du travail, du profit, de l'entreprise, etc., et les mêmes types d'objectifs sociaux (indépendance, développement, transmission, etc.) et économiques (produire, développer,faire des profits, etc.), développent les mêmes logiques d'action et les mêmes comportements et choix économiques. Les micromentalités qui renvoient à un niveau subjectif de l'analyse seront confrontées au fonctionnement concret des petites entreprises (formes d'organisations, orientations stratégiques et démarches marketing) pour vérifier leur efficacité et leur capacité explicative.

Les micromentalités, propres à des groupes restreints, forment donc des visions globales de l'économie et de l'activité professionnelle et produisent des logiques d'action pragmatiques. En effet, respectivement, les activités économique et professionnelle ne sont pas extraites de la vie sociale des entrepreneurs dirigeants et leurs actions peuvent intégrer des éléments contradictoires (p. ex. profit et passion). Elles sont en effet susceptibles d'orienter les comportements des entrepreneurs et, en cela, elles sont efficaces. Leur efficacité s'exprimerait alors par les logiques pratiques de l'action qu'elles produisent. Ces dernières ne sont pas systématiquement conformes aux rationalités théoriques ou aux modes de pensée des virtuoses de l'économie (Gislain et Steiner, 1995).

L'usage des micromentalités qui s'inscrit dans une démarche inductive et empirique ne vise pas à produire une analyse forcément conforme aux canons de l'économie ou de la gestion rationnelle des entreprises. Elle se fixe comme objectif l'analyse des comportements économiques «pratiques» des entrepreneurs tels qu'ils se déroulent sans aucun préjugé. Le but étant de comprendre le fonctionnement réel de l'économie des PE-TPE d'un secteur particulier. En d'autres termes, le concept de micromentalités part de l'idée que se font les entrepreneurs dirigeants de l'économie et de leur activité professionnelle pour expliquer leurs comportements et non pas de l'idée que se fait l'économie de «l'entrepreneur dirigeant idéal». 


\section{Opérationalisation du concept de micromentalité : Le cas des PE-TPE du tourisme sportif}

Les résultats de la recherche qui a concerné les PE-TPE du tourisme sportif révèlent que les micromentalités orientent les actions économiques des dirigeants et participent à produire des modes de gestions spécifiques, et ce, en privilégiant certains buts et motifs inhérents aux visions subjectives qu'ont les entrepreneurs du mode économique et professionnel (Bouhaouala, 1999). En somme, les micromentalités ( $c f$.figure 1), déterminent des rapports particuliers au profit, au travail, à l'entreprise, à la concurrence, à la réussite sociale, au sport, et produisent des logiques d'action propres à des groupes d'entrepreneurs dirigeants et modes de gestion.

\section{Figure 1}

\section{Modèle d'analyse}

\begin{tabular}{|lll|}
\hline \multicolumn{2}{|c|}{ Micromentalité des entrepreneurs dirigeants } \\
& & \\
Conception & Valeurs & Objectifs \\
- Travail, entreprise & - Économiques & - Financiers \\
- Concurrence, marché & - Sociales & - Sociaux \\
- Sport, profit & - Professionnelles & - Professionnels \\
& & \\
\hline
\end{tabular}

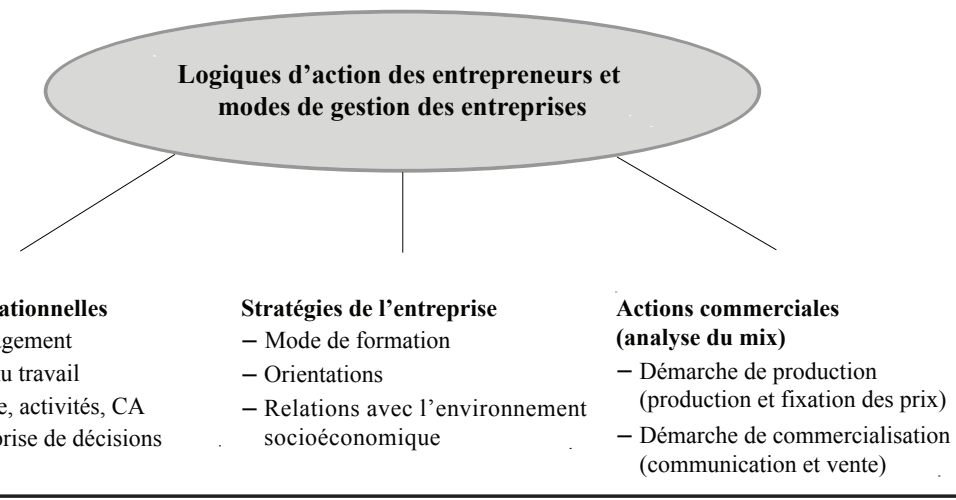

Revue internationale P.M.E., vol. 20, n 2, 2007 
L'application du concept de micromentalité à l'analyse de l'univers des PE-TPE du tourisme sportif a mis en évidence une typologie taxonomique ${ }^{2}$ des entrepreneurs dirigeants et de leurs entreprises: 1) l'indépendant passionné (IP), 2) l'entrepreneur indépendant (EI), 3) le manager gestionnaire (MG) et 4) le conservateur patrimonial (Bouhaouala, 1999).

La reconstruction des logiques «pratiques» d'action des entrepreneurs dirigeants a fourni des éléments de connaissance pertinents pour comprendre le degré de complexité du fonctionnement des PE-TPE du tourisme sportif et notamment pour identifier les connexions possibles entre les dimensions économiques et non économiques dans les modes de gestion, la prise de décisions... ainsi que dans les comportements concurrentiels (Bouhaouala, 2001) et de pratiques commerciales (Bouhaouala et Chantelat, 2002) des PE-TPE. En d'autres termes, les logiques d'actions spécifiques ont montré des écarts et contradictions dans les choix des dirigeants et au niveau des comportements des petites entreprises par rapport à la rationalité économique.

\section{Typologie taxonomique des entrepreneurs et des PE-TPE}

Avant de présenter la typologie taxonomique des entrepreneurs et des entreprises, il convient de préciser le cadre d'utilisation de cet outil méthodologique. Si l'on se réfère aux définitions des classifications (idéal-typologie et taxonomie), on arrive à les distinguer par rapport à l'approche adoptée et à leur relation au modèle théorique de référence (Doty et Glick, 1994). Mais d'une façon générale, les classifications ont pour objectif de rendre le réel, complexe et chaotique à l'origine, intelligible et compréhensible (Miller, 1996). La typologie s'inscrit davantage dans les approches déductives cherchant à définir les variables prédéterminées théoriquement afin de constituer une classification explicative. La taxonomie quant à elle s'inscrit dans les approches inductives et empiriques cherchant à orchestrer les caractéristiques les plus significatives pour établir une classification compréhensible (Candau, 1981). Le lien entre le modèle théorique de référence n'est pas à démontrer, mais c'est le sens de ce lien qui fait la différence entre l'idéal-typologie et la taxonomie. Pour conclure cette précision, on peut dire que la typologie taxonomique est une classification inductive multicritère testée empiriquement en rapport avec le modèle théorique sans que ce dernier ne prédétermine une quelconque orientation. Ce travail s'inscrit dans une approche empirique et inductive qui a consisté à reconstruire les micromentalités des entrepreneurs et à identifier les caractéristiques les plus discriminantes dans

2. La construction de la typologie taxonomique des entrepreneurs dirigeants et des petites entreprises est fondée sur l'identification de: 1) micromentalités spécifiques, 2) choix organisationnels, 3) stratégies commerciales, 4) démarches marketing et, enfin, de 5) logiques d'action. 
le fonctionnement des PE-TPE. La confrontation des deux niveaux d'analyse subjectif (micromentalités) et objectif (caractéristiques des PE-TPE) a permis de faire émerger une typologie taxonomique démontrant l'efficacité et la force explicative du modèle théorique fondé sur les micromentalités des entrepreneurs.

\section{La microentreprise unipersonnelle de l'indépendant passionné (IP)}

Les microentreprises des indépendants passionnés sont souvent jugées comme étant insuffisamment performantes à cause de leur très petite taille et de leur mode de gestion. En effet, leurs dirigeants adoptent des comportements déviants par rapport à la recherche systématique de la maximisation des profits. Mais au vu de leur stratégie et de leur fonctionnement, ils ne sont pas si irrationnels que le laisserait entendre une analyse économique pure. En effet, leurs choix économiques et organisationnels sont orientés par une logique d'action hédoniste qui est en adéquation avec leur micromentalité de passionnés pour laquelle le sport-passion est une finalité; l'entreprise et le profit sont des moyens au service de l'assouvissement de leur passion. La concurrence, par exemple, est ignorée; elle n'existe pas à leurs yeux.

Les TPE des IP qui fonctionnent selon cette logique hédoniste se caractérisent par:

1) Un mode de management organique et une démarche productive centrée sur «soi» (passion personnelle). Cela produit une forte «spécialisation de la TPE» dans un service sportif qui se traduit, le plus souvent, par une forte «personnalisation» des produits et par un «rapport fusionnel» à une clientèle partageant les mêmes valeurs et qui recherche un type de services original et marginal éloigné des standards du marché. En effet, le maintien de l'activité de l'entreprise est rendu possible par la forte spécialisation et la concentration sur une clientèle à fort pouvoir d'achat;

2) la résistance aux contraintes et tendances universelles du marché (rentabilité, concurrence, tendance à la croissance, concentration, alliance, etc.). En effet, les IP résistent aussi bien aux conjonctures difficiles qu'aux périodes fastes. Ils maintiennent leur entreprise et leur volume d'activités au niveau qui leur assure l'indépendance et l'assouvissement de leur passion face aux pressions économiques, velléités de rachat et tendances de croissance. Leurs liberté et indépendance reposent en contrepartie sur le refus de la croissance et de l'agrandissement de l'entreprise qui signifient pour eux une dépendance à l'égard des marchés du travail et des capitaux. Autrement

Revue internationale P.M.E., vol. 20, n 2, 2007 
dit, par leur résistance aux orientations et contraintes économiques du marché, les IP démontrent qu'ils ont su développer des stratégies performantes à leur niveau. L'âge de leur entreprise dépassant les 10 ans constitue une preuve supplémentaire de leur performance stratégique et commerciale.

\section{Synthèse multicritère de la microentreprise de l'indépendant passionné}

\begin{tabular}{ll}
\hline PRINCIPES ORGANISATEURS & DIMENSIONS DE LA TYPOLOGIE \\
\hline Micromentalité & Indépendant passionné
\end{tabular}

1. Valeurs

2. Objectif social et économique

3. Conception de la réussite

4. Conception du travail

5. Conception de l'entreprise

6. Conception de l'argent

7. Conception du sport
1. Passion du sport, indépendance, sobriété.

2. Vivre de sa passion toute l'année. Recherche du supplément d'être et non pas de l'avoir.

3. Atteindre la qualité de la vie escomptée.

4. Partage de sa passion, sans patron, a une fonction «alimentaire», c'est un moyen pour vivre.

5. Contrainte de travail et de rentabilité, lieu d'aliénation, soumission au marché.

6. A une fonction uniquement sociale, c'est un moyen et non une finalité.

7. Le sport est: passion, liberté et plaisir.

\section{Forme organisationnelle}

1. Type de management

2. Modalité d'accès à la direction

3. Rapport à l'embauche

4. Activité principale commercialisée

5. Noyau dur de l'activité du dirigeant

6. La famille du dirigeant

7. Statut juridique de l'organisation

8. Taille (chiffre d'affaires et effectif)

\section{Microentreprise unipersonnelle}

1. Organique.

2. Création ex nihilo.

3. Pas d'embauche: risque de dénaturer la souplesse du fonctionnement et affaiblir son indépendance.

4. Encadrement d'un service sportif par saison.

5. Production et encadrement de services sportifs; tâches administratives.

6. Implication de la famille dans le fonctionnement.

7. Entreprise unipersonnelle (travailleur indépendant).

8. TPE: chiffre d'affaires $<$ à $75 \mathrm{~K} €$ et 0 salarié. 


\begin{tabular}{|c|c|}
\hline PRINCIPES ORGANISATEURS & DIMENSIONS DE LA TYPOLOGIE \\
\hline Stratégie & Implicite de spécialiste \\
\hline 1. Mode de formation des stratégies & $\begin{array}{l}\text { 1. Implicite selon une «idéologie } \\
\text { passionnelle». }\end{array}$ \\
\hline 2. Orientation stratégique & $\begin{array}{l}\text { 2. Centrée sur l'activité sportive en tant } \\
\text { que passion personnelle du dirigeant. }\end{array}$ \\
\hline 3. Type de stratégie & $\begin{array}{l}\text { 3. Diversification hiver/été imposée } \\
\text { par rapport au projet du dirigeant et } \\
\text { spécialisation sportive «naturelle» } \\
\text { par rapport à sa passion. }\end{array}$ \\
\hline 4. Atout stratégique & $\begin{array}{l}\text { 4. Haute expertise sportive et parfaite } \\
\text { connaissance du territoire local. }\end{array}$ \\
\hline 5. Réseaux & $\begin{array}{l}\text { 5. Territoriaux/informels et extrater- } \\
\text { ritoriaux/formels basés sur les liens } \\
\text { sociaux, sur le partage d'une vision } \\
\text { passionnelle et authentique du } \\
\text { tourisme sportif. }\end{array}$ \\
\hline
\end{tabular}

\section{Marketing}

1. Production

2. Formation des prix

3. Communication

4. Commercialisation

\section{Démarche artisanale centrée sur la passion}

1. Démarche orientée sur soi et sa passion.

2. Estimation subjective, honoraires calculés indépendamment des coûts de production.

3. Non nécessaire fondée sur le bouche à oreille.

4. Fondée sur un rapport fusionnel et affectif aux clients (clientèle fidélisée).

\section{Logique d'action}

\section{Hédoniste}

Les IP perçoivent l'entreprise comme un moyen de garantir leur liberté, d'assouvir leur passion du sport et non pas comme une fin en soi. Ils développent des choix et des comportements cohérents avec une logique d'action hédoniste tout en composant avec la réalité économique et sociale intégrant ainsi des éléments contradictoires (être libre et gagner sa vie). En effet, leur logique n'est pas en adéquation avec celle de l'univers marchand duquel ils dépendent professionnellement, économiquement et socialement. En ce sens, ils font des compromis avec la réalité économique et sociale sans pour autant devenir des prestataires de services sportifs à vocation purement commerciale (pour plus de détails, $c f$. tableau 1). 


\section{L'Agence des sports outdoor de l'entrepreneur indépendant (EI)}

Par sa soif de croissance et d'indépendance, l'entrepreneur indépendant se rapproche du modèle des «nouveaux entrepreneurs » (Julien et Marchesnay, 1988); par l'importance qu'il accorde à la production (création, amélioration, innovation, etc.), au développement de ses activités; par sa négligence de la dimension patrimoniale de l'entreprise, il se rapproche aussi du «génial technicien» (Bauer, 1993), et par sa recherche d'expansion des activités, de rentabilité sur la longue durée (des produits et de l'entreprise) et des bonnes affaires, il peut être qualifié d'opportuniste ou rapproché de «l'entrepreneur capitaliste» (Gresle, 1981; Zarca, 1986).

Cela dit, bien que ces caractéristiques soient présentes dans les comportements de l'EI, ces rapprochements sont à nuancer. Dans le sens que c'est sa logique d'action entrepreneuriale et individualiste qui engendre des effets économiques. En effet, la réussite de sa propre entreprise, s'exprimant par des grandeurs économiques (rentabilité, parts de marché, croissance, investissement, etc.), n'est qu'un indicateur de son objectif principal: la réalisation de soi en tant qu'individu social à travers l'action entrepreneuriale.

Cependant, l'importance de ces effets économiques ne prend du «sens» qu'aux yeux de l'entrepreneur lui-même. Ce dernier, comme le self-made man parti de rien ne compte que sur «soi». Il a réussi à créer sa propre entreprise en mobilisant son propre capital et ses convictions; compétences et expériences personnelles, etc. L'estimation de la réussite de son entreprise et de ses effets économiques prend son importance davantage sur le plan «subjectif» qu'objectif. Autrement dit, l'importance subjective de la réussite sociale peut être en décalage avec sa valeur économique réelle, car elle est parfois hypertrophiée. 
Tableau 2

\section{Synthèse multicritère de l'Agence des sports} de l'entrepreneur indépendant

\begin{tabular}{l}
\hline PRINCIPES ORGANISATEUR \\
Micromentalité \\
1. Valeurs \\
2. Objectif social et économique \\
3. Conception de la réussite \\
4. Conception du travail \\
5. Conception de l'entreprise \\
6. Conception de l'argent \\
7. Conception du sport
\end{tabular}

Forme organisationnelle

1. Type de management

2. Modalité d'accès à la direction

3. Rapport à l'embauche

4. Activités principales commercialisées

5. Noyau dur de l'activité du dirigeant

6. La famille du dirigeant

7. Statut juridique de l'organisation

8. Taille (chiffre d'affaires et effectif)

\section{DIMENSIONS DE LA TYPOLOGIE}

\section{Entrepreneur indépendant}

1. Indépendance, créativité, ténacité.

2. Réalisation de soi et développement de l'entreprise.

3. Entreprendre avec succès et indépendance.

4. Entreprendre, faire des efforts, être créatif.

5. Finalité, objet d'accomplissement de soi, lieu de création.

6. Instrument économique et signe de réussite sociale

7. Service monnayable, potentialités commerciales.

\section{Agence des sports outdoor}

1. Empirique centralisé.

2. Création seul ou en association avec des amis.

3. Embauche de personnel compétent (nécessaire pour le développement de l'entreprise) sans perte de pouvoir.

4. Encadrement, services sportifs et organisation d'événements.

5. Production de services et relation au client.

6. Tenue à l'écart du fonctionnement, mais impliquée dans le capital de l'entreprise.

7. Entreprise unipersonnelle et SARL.

8. PE-TPE: chiffre d'affaires: 150 à $750 \mathrm{~K} €, 3$ à 14 salariés. 


\begin{tabular}{|c|c|}
\hline PRINCIPES ORGANISATEURS & DIMENSIONS DE LA TYPOLOGIE \\
\hline Stratégie & Empirique de diversification de services \\
\hline 1. Mode de formation des stratégies & $\begin{array}{l}\text { 1. Explicite empirique selon } \\
\text { «l'idéologie de l'entrepreneur». }\end{array}$ \\
\hline 2. Orientation stratégique & $\begin{array}{l}\text { 2. Orientée sur l'innovation, le produit } \\
\text { et les clients. }\end{array}$ \\
\hline 3. Type de stratégie & $\begin{array}{l}\text { 3. Diversification des services avec } \\
\text { ciblage d'un segment du marché } \\
\text { ou spécialisation sportive avec } \\
\text { diversification de marché. }\end{array}$ \\
\hline 4. Atout stratégique & $\begin{array}{l}\text { 4. Compétences techniques et } \\
\text { commerciales avec une bonne } \\
\text { connaissance du marché. }\end{array}$ \\
\hline 5. Réseaux & $\begin{array}{l}\text { 5. Informels locaux et nationaux basés } \\
\text { sur des liens commerciaux et des } \\
\text { intérêts économiques. }\end{array}$ \\
\hline Marketing & $\begin{array}{l}\text { Démarche entrepreneuriale centrée } \\
\text { sur le produit }\end{array}$ \\
\hline 1. Production & $\begin{array}{l}\text { 1. Poussée par l'innovation technique } \\
\text { (sportive) et technologique. }\end{array}$ \\
\hline 2. Formation des prix & $\begin{array}{l}\text { 2. Estimation empirique fondée sur les } \\
\text { coûts de production classiques. }\end{array}$ \\
\hline 3. Communication & $\begin{array}{l}\text { 3. Correspond à l'objectif de l'entrepre- } \\
\text { neur, externalisée en grande partie. }\end{array}$ \\
\hline 4. Commercialisation & $\begin{array}{l}\text { 4. Partagée entre vente directe et inter- } \\
\text { médiaire (agences et réseaux). }\end{array}$ \\
\hline Logique d'action & Entrepreneuriale et individualiste \\
\hline
\end{tabular}

De ce fait, la dimension individuelle est centrale dans sa logique d'action pour une double raison: la première, bien que l'action entrepreneuriale produise des effets socioéconomiques sur l'environnement (emplois, activités, etc.), elle reste exclusivement centrée sur les motifs et projets personnels de l'entrepreneur; la deuxième est que la réussite ne sera satisfaisante que dans la mesure où «il y arrive seul contre obstacles et embûches ». Ce n'est qu'ainsi qu'il se sent «bien exister» et accompli.

L'indépendance de l'EI est obtenue par le contrôle du capital de l'entreprise (grâce à la participation de la famille et des proches) et par sa capacité à détourner les paramètres du marché en sa faveur (nouveaux produits, nouveaux marchés, anticipation, réseaux, etc.). L'EI a opté pour la forme de l'Agence des sports outdoor qui se caractérise donc par un mode de management centralisé et par une démarche productive entrepreneuriale axée sur la valorisation des innovations et la diversification des services sportifs et des marchés. 
L'EI se distingue donc de l'entrepreneur capitaliste par le fait que sa logique d'action est parfois en décalage par rapport à celle du système capitaliste ou du monde marchand. Son objectif réel est social, même s'il prend une «couleur» économique. De ce fait, ses relations avec l'environnement socioéconomique (le marché, les institutions financières, les organismes publics de promotion et de développement du tourisme, etc.) ne s'inscrivent pas toujours dans un rapport de coopération économique.

En somme, les entrepreneurs indépendants, bien que produisant des effets économiques importants sur le marché, ne réagissent pas mécaniquement aux paramètres de ce dernier comme le laisserait entendre le modèle économique de l'homo æeconomicus, mais agissent suivant la logique de l'entrepreneur individualiste.

\section{L'organisation sociomanagériale du manager gestionnaire (MG)}

Le MG, contrairement aux autres dirigeants, est salarié de l'organisation. Son «esprit d'entreprise» n'est pas celui du «capitaliste» ou de l'entrepreneur indépendant décrit plus haut. En effet, il se distingue de ces deux modèles par son besoin «d'être utile socialement» (à son environnement proche et local). Sa capacité d'entreprendre est en quelque sorte mise à disposition de projets socioéconomiques collectifs ou locaux (tourisme social, développement local, etc.).

Le modèle de l'entreprise qu'il dirige ressemble à celui de «l'entreprise alternative » décrite par Joyal (1986) et son mode de fonctionnement est fondé sur une idéologie à la fois sociétale et managériale. Dans sa vision du monde économique et professionnel, bien que les objectifs visés par l'entreprise (économiques et/ou sociaux) dépendent du marché, l'entreprise est perçue comme étant au service d'un projet sociétal. En d'autres termes, le MG accorde une vocation collective et sociétale même aux fonctions économique et commerciale de l'entreprise.

Les valeurs qui fondent la micromentalité des MG sont des valeurs de partage social (dévouement, responsabilité, solidarité, engagement, etc.). De ce fait, l'entreprise est perçue comme un outil au service de l'environnement social. Les dirigeants lui accordent alors une fonction sociale qui dépasse les limites de son activité commerciale.

Les objectifs économiques de l'entreprise sont partagés par les dirigeants et ne sont pas perçus comme contradictoires avec leurs objectifs sociaux. En effet, le principal objectif des dirigeants est d'être socialement utile; ils ont l'impression de réussir dans leur travail lorsqu'ils «créent pour 
les autres » et lorsqu'ils assurent leurs engagements envers l'entreprise. Leur indépendance relative d'action est donc due à la dimension morale qu'ils perçoivent dans leur fonction (responsabilité envers l'organisation).

L'entreprise est plus qu'un employeur effectif ou potentiel. Pour eux, c'est un outil de développement socioéconomique, l'activité et la richesse qu'elle produit ne sont que des effets économiques nécessaires à la réalisation de sa fonction sociale. En effet, bien qu'ils croient que le marché est un univers fonctionnant selon les règles rationnelles de l'économie (rentabilité, efficacité, croissance, compétitivité, etc.), ils sont aussi convaincus que le marché, autant que l'entreprise, doit rester au service d'objectifs sociétaux (créer des emplois, rendre le tourisme sportif accessible, etc.).

De ce fait, leur logique d'action est fondée sur deux dimensions: sociale et managériale. La première, par ses objectifs presque sacrés, justifie la seconde, c'est-à-dire la rigueur et la rationalité managériale avec lesquelles ils dirigent l'entreprise. D'une autre manière, la seconde est au service de la première et, en cela, l'une relativise l'autre pour aller vers une logique d'action sociomanagériale.

L'entreprise des managers gestionnaires se caractérise alors par 1) un mode de gestion sociétal et managérial hiérarchisé avec une rationalisation du processus de prise de décision (conseil d'administration, direction, etc.) et par 2) une démarche productive fondée sur la spécialisation du personnel, axée sur le client et le marché en vue de la réalisation des objectifs socioéconomiques.

Le marché est alors considéré comme un monde social dans lequel ils ont pour mission de trouver «la logique alternative» à la rationalité purement marchande (le profit maximum avant tout). Bien que leur logique d'action intègre en partie l'idéologie managériale, par sa dimension sociale ou «sociétale», elle est sur le fond en porte-à-faux par rapport à la logique du monde marchand. Cela dit, cette logique sociomanagériale rend possible l'adoption d'un mode de gestion qui permet de faire tenir ensemble deux logiques parfois contradictoires et, donc, de coopérer avec les environnements sociaux, institutionnels (politique, publique, parapublique) et économiques pour atteindre leurs objectifs originels. 


\section{TABLeau 3}

\section{Synthèse multicritère de l'Organisation sociomanagériale} du manager gestionnaire

\begin{tabular}{l}
\hline PRINCIPES ORGANISATEUR \\
\hline Micromentalité \\
1. Valeurs \\
2. Objectif social et économique \\
3. Conception de la réussite \\
4. Conception du travail \\
5. Conception de l'entreprise \\
6. Conception de l'argent \\
7. Conception du sport
\end{tabular}

\section{Forme organisationnelle}

1. Type de management

2. Modalité d'accès à la direction

3. Rapport à l'embauche

4. Activité principale commercialisée

5. Noyau dur de l'activité du dirigeant

6. La famille du dirigeant

7. Statut juridique de l'organisation

8. Taille (chiffre d'affaires et effectif)

\section{DIMENSIONS DE LA TYPOLOGIE}

Manager gestionnaire

1. Rigueur, engagement, dévouement, responsabilité.

2. Être utile socialement, maintien et développement de l'entreprise.

3. Créer et maintenir des emplois, agir socialement.

4. Autonomie relative, assumer ses responsabilités, efficacité.

5. Outil de développement socioéconomique local.

6. Fonction sociale importante.

7. Atout commercial, produit commercial.

\section{Organisation sociomanagériale}

1. Rationnel, sociétal et managérial.

2. Par salariat.

3. Sociétal, fondé sur les liens locaux; managérial fondé sur les compétences nécessaires.

4. Séjours en centres de vacances comprenant: hébergement, restauration, animation sportive et divers services annexes (garderie, location de matériel, etc.).

5. Centrée sur la gestion et l'organisation de l'entreprise.

6. Aucune relation avec le fonctionnement de l'entreprise.

7. SA, SEM et Entreprise associative (loi 1901).

8. PE et TPE: chiffre d'affaires de $230 \mathrm{~K} €$ à $3,35 \mathrm{M} €, 3$ à 50 salariés. 


\begin{tabular}{|c|c|}
\hline PRINCIPES ORGANISATEURS & DIMENSIONS DE LA TYPOLOGIE \\
\hline Stratégie & Managériale d'intégration horizontale \\
\hline 1. Mode de formation des stratégies & $\begin{array}{l}\text { 1. Formalisé et rationalisé selon } \\
\text { «l'idéologie managériale». }\end{array}$ \\
\hline 2. Orientation stratégique & $\begin{array}{l}\text { 2. Orientée sur le marché à visée } \\
\text { commerciale. }\end{array}$ \\
\hline 3. Type de stratégie & $\begin{array}{l}\text { 3. Intégration horizontale des services } \\
\text { avec concentration sur un type de } \\
\text { marché. }\end{array}$ \\
\hline 4. Atout stratégique & $\begin{array}{l}\text { 4. Importante capacité d'accueil, } \\
\text { réseaux de commercialisation bien } \\
\text { développé et spécialisé. }\end{array}$ \\
\hline 5. Réseaux & $\begin{array}{l}\text { 5. Formels nationaux basés sur des liens } \\
\text { commerciaux et sociétaux. }\end{array}$ \\
\hline Marketing & $\begin{array}{l}\text { Démarche managériale centrée } \\
\text { sur le marché }\end{array}$ \\
\hline 1. Production & $\begin{array}{l}\text { 1. Tirée par le marché, la concurrence } \\
\text { et le segment ciblé. }\end{array}$ \\
\hline 2. Formation des prix & $\begin{array}{l}\text { 2. Selon les coûts de production, la } \\
\text { concurrence et le pouvoir d'achat } \\
\text { des clients. }\end{array}$ \\
\hline 3. Communication & $\begin{array}{l}\text { 3. Externalisée avec ciblage de } \\
\text { clientèles à une échelle nationale. }\end{array}$ \\
\hline 4. Commercialisation & $\begin{array}{l}\text { 4. Essentiellement externalisée par } \\
\text { des réseaux à force de vente élevée. }\end{array}$ \\
\hline Logique d'action & Sociomanagériale \\
\hline
\end{tabular}

\section{L'entreprise familiale du conservateur patrimonial (CP)}

L'entreprise du CP est familiale et fonctionne selon une logique d'action conservatrice et patrimoniale. Les caractéristiques de son mode de gestion et fonctionnement révèlent un manque d'action entrepreneuriale qui rapproche le CP du modèle de «l'occupant de la maison familiale» (Bauer, 1993); sa méfiance à l'égard du marché du travail et des capitaux le rapproche de «l'artisan» (Gresle, 1981); l'objectif d'indépendance familiale le rapproche de «l'entrepreneur PIC» (Julien et Marchesnay, 1988) et, enfin, la recherche, par le dirigeant, de l'accumulation du capital le rapproche de «l'entreprise artisanale capitaliste» (Zarca, 1986).

Dans la micromentalité du CP, la prédominance de la dimension patrimoniale se traduit par des conceptions particulières de l'entreprise, du marché et par des objectifs contradictoires. En effet, la volonté morale de pérenniser 
l'entreprise dans le patrimoine familial et la volonté économique de faire des profits financiers à court terme produisent un mode de gestion traditionaliste. Le dirigeant de ce type d'entreprise est plus conservateur qu'entrepreneur.

L'objectif de vouloir conserver absolument ce qui existe et de continuer à vivre le passé prospère des générations antérieures s'inscrit dans une vision traditionaliste et conservatrice. Le bénéfice, le profit, la réussite financière, etc., sont des effets économiques souhaités, mais non engendrés par un projet entrepreneurial. L'entreprise est un capital familial qui constitue une rente et une source de revenus à conserver absolument.

L'argent et l'entreprise sont à la fois une finalité et un moyen, c'est-àdire respectivement ils représentent un aboutissement des efforts familiaux et un moyen d'accumulation du capital par l'investissement dans le patrimoine familial. Cela rejoint l'idée que la recherche d'indépendance n'est pas «inconciliable» avec l'esprit du capitalisme (Gresle, 1981).

Par ailleurs, il ressort de l'analyse de leur démarche productive «traditionaliste» une inadéquation entre leur finalité économique et la manière dont ils veulent l'atteindre. On a observé une absence de prise de risque et d'innovation industrielle ou commerciale. Cela indique que ce n'est pas leur recherche de la performance économique qui détermine leurs comportements et choix stratégiques, mais plutôt leur «vision patrimoniale de l'entreprise ». Leur logique d'action est donc constituée d'une contradiction entre la finalité économique et la finalité sociale. La seconde limite en grande partie les moyens de la première, il y a donc un tiraillement entre leur «rationalité matérielle» et leur «rationalité formelle»(Weber, 1964).

En effet, leur dimension patrimoniale réduit leur stratégie économique au niveau d'une réaction (lente) in extremis dans le but d'éviter la disparition ou la prise de contrôle de l'entreprise par des tiers (financiers, employés, etc.). En d'autres termes, les CP réagissent en investissant dans le but de conserver leur mode de vie et de travail; leur rente et leur patrimoine. Cela signifie qu'ils n'agissent pas dans une perspective de développement et de croissance, mais de conservation et de survie.

Cela s'explique par le fait qu'ils vivent le marché dans l'espoir que le passé prospère (avant les années 1980) de leurs parents se reproduise, et ce, sans prise de risque. Ces derniers, à l'origine propriétaires terriens bien implantés dans le territoire depuis plusieurs générations, avaient effectivement vécu sans grands efforts commerciaux les vagues successives du grand «boom » du tourisme (d'après-guerre:1945). Leur mérite était d'être là et de profiter de la situation en changeant d'activité professionnelle et en adaptant leurs fermes au tourisme. Parce que leurs familles sont bien ancrées territoria- 
lement, ils considèrent que le marché local fait partie du patrimoine culturel et économique familial. Autrement dit, l'espace territorial est leur source de richesse, les «bons touristes » doivent donc continuer à payer «correctement » leur passage. Leur rapport au client est fondé sur des relations d'échanges commerciaux et de paiement d'un droit de séjour.

Les CP ne voient pas leur manque d'ouverture à l'embauche de compétences ou de capitaux extérieurs à la famille comme des freins à leurs objectifs économiques, mais le perçoivent plutôt comme un moyen de réaliser leur indépendance. Cette dernière est garantie par la prise en charge familiale du travail et par son implication dans le capital (répartition des tâches et des responsabilités, prise de décision, constitution du capital, etc.). La logique conservatrice et patrimoniale accorde donc une position centrale à la famille et engendre un manque de coopération avec l'environnement socioéconomique.

L'entreprise des CP se caractérise alors par 1) un mode de management familial paternaliste déterminé en grande partie par la responsabilité du pater familias de conserver et de transmettre le patrimoine (entreprise et savoir-faire) et 2) une démarche productive inadéquate avec leur "fin» (maximisation des gains) à court terme se rapprochant de celle du « commerçant classique ». Cette dernière se résume dans une démarche traditionaliste visant le maintien de la part de marché déjà acquise et manque de prise de risque, d'innovation et de créativité.

Leur logique d'action est donc en décalage avec les «lois rationnelles du management ». Fondée sur des éléments contradictoires, elle est ambivalente, se situant entre la volonté de faire du commerce et le conservatisme. Le CP rejoint en cela «l'occupant de la maison familiale» (Bauer, 1993) et «l'artisan non dynamique et attentiste» (Mardellat, 1994). De peur de ne pas réussir sur le plan moral et social, c'est-à-dire de conserver l'entreprise telle qu'elle a été transmise ou en meilleure situation financière, le $\mathrm{CP}$, répulsé par l'incertitude, ne fera que ce qui est conforme à la tradition, c'est-à-dire «sûr et rassurant » économiquement. En ce sens, il est à l'opposé de l'entrepreneur agissant sans hésitation et même profitant de l'incertitude pour améliorer sa situation (Julien et Marchesnay, 1996). 


\section{TABLEAU 4}

Synthèse multicritère de l'entreprise familiale du conservateur patrimonial

PRINCIPES ORGANISATEURS DIMENSIONS DE LA TYPOLOGIE

Micromentalité

1. Valeurs

2. Objectif social et économique

3. Conception de la réussite

4. Conception du travail

5. Conception de l'entreprise

6. Conception de l'argent

7. Conception du sport

Forme organisationnelle

1. Type de management

2. Modalité d'accès à la direction

3. Rapport à l'embauche

4. Activité principale commercialisée

5. Noyau dur de l'activité du dirigeant

6. La famille du dirigeant

7. Statut juridique de l'organisation

8. Taille (chiffre d'affaires et effectif)
Conservateur patrimonial

1. Sacrifice, loyauté à la famille et à la tradition, indépendance.

2. Maintenir l'entreprise dans la famille et assurer les bénéfices à court terme.

3. Gagner de l'argent et transmettre l'entreprise.

4. Savoir-faire familial, moyen de réussir et de préserver l'indépendance de l'entreprise.

5. Patrimoine familial et source de richesse.

6. Rente pour la famille, investissement dans le patrimoine.

7. Force d'attractivité commerciale.

\section{Entreprise familiale}

1. Familial et paternaliste.

2. Par transmission (héritage ou acquisition en famille).

3. Pas de salariés extérieurs à la famille sur des postes clés.

4. Services d'accueil et/ou services sportifs traditionnels (ski de fond, randonnée pédestre).

5. Centrée sur la gestion et la production.

6. Fortement impliquée dans le fonctionnement et le capital de l'entreprise.

7. EURL, SARL, SA.

8. PE et TPE: chiffre d'affaires de $75 \mathrm{k} €$ à $1,5 \mathrm{M} €$, de 1 à 25 salariés. 


\begin{tabular}{|c|c|}
\hline PRINCIPES ORGANISATEURS & DIMENSIONS DE LA TYPOLOGIE \\
\hline Stratégie & Traditionaliste \\
\hline 1. Mode de formation des stratégies & $\begin{array}{l}\text { 1. Traditionaliste selon «l'idéologie } \\
\text { du commerçant patrimonial». }\end{array}$ \\
\hline 2. Orientation stratégique & $\begin{array}{l}\text { 2. Orientée sur le maintien du capital } \\
\text { familial et de la rente. }\end{array}$ \\
\hline 3. Type de stratégie & $\begin{array}{l}\text { 3. Positionnement sur un segment } \\
\text { avec diversification dans les services } \\
\text { traditionnels (hébergement et } \\
\text { animation sportive). }\end{array}$ \\
\hline 4. Atout stratégique & $\begin{array}{l}\text { 4. Ancienneté du savoir-faire } \\
\text { et sur le marché local. }\end{array}$ \\
\hline 5. Réseaux & $\begin{array}{l}\text { 5. Informel local; formel et commercial } \\
\text { national. }\end{array}$ \\
\hline
\end{tabular}

\section{Marketing}

1. Production

2. Formation des prix

3. Communication

4. Commercialisation

\section{Démarche traditionaliste centrée sur le savoir-faire familial}

1. Selon le savoir-faire traditionaliste; les intermédiaires et la clientèle.

2. Selon les coûts de production, la clientèle et les conventions locales.

3. Externalisée, car ne fait pas partie du métier.

4. Essentiellement par des réseaux intermédiaires.

\section{Logique d'action}

Conservatrice et patrimoniale

\section{Conclusion}

Cette typologie multicritère s'appuyant sur le concept de micromentalité des dirigeants et des logiques d'action qui en découlent a mis en évidence des modes de gestion spécifiques (formes organisationnelles, démarches stratégiques et productives) qu'une typologie quantitative ou déterministe n'aurait pas permis de révéler.

Il est évident que le choix préalable de tel ou tel critère objectif comme base de l'analyse (lié à l'entreprise ou au dirigeant) aurait conduit soit à des typologies simples et limitées à des extrêmes, soit à des découpages produisant un grand nombre de sous-types de PE-TPE et de dirigeants (Bentabet, Michun et Trouvé, 1999; Torrès, 1998). Par exemple, si l'axe de partage choisi était le mode d'accès à la position de dirigeant (création, embauche ou transmission), le statut juridique, la taille, l'activité principale, etc., les typologies seraient bipolaires, voire tripolaires, mais dans tous les cas, elles seraient incapables de rendre compréhensibles les différences réelles de modes de gestion, de production et d'organisation des PE-TPE ( $c f$. figure 2). 
Figure 2

\section{Simulation de typologie à partir de données objectives}

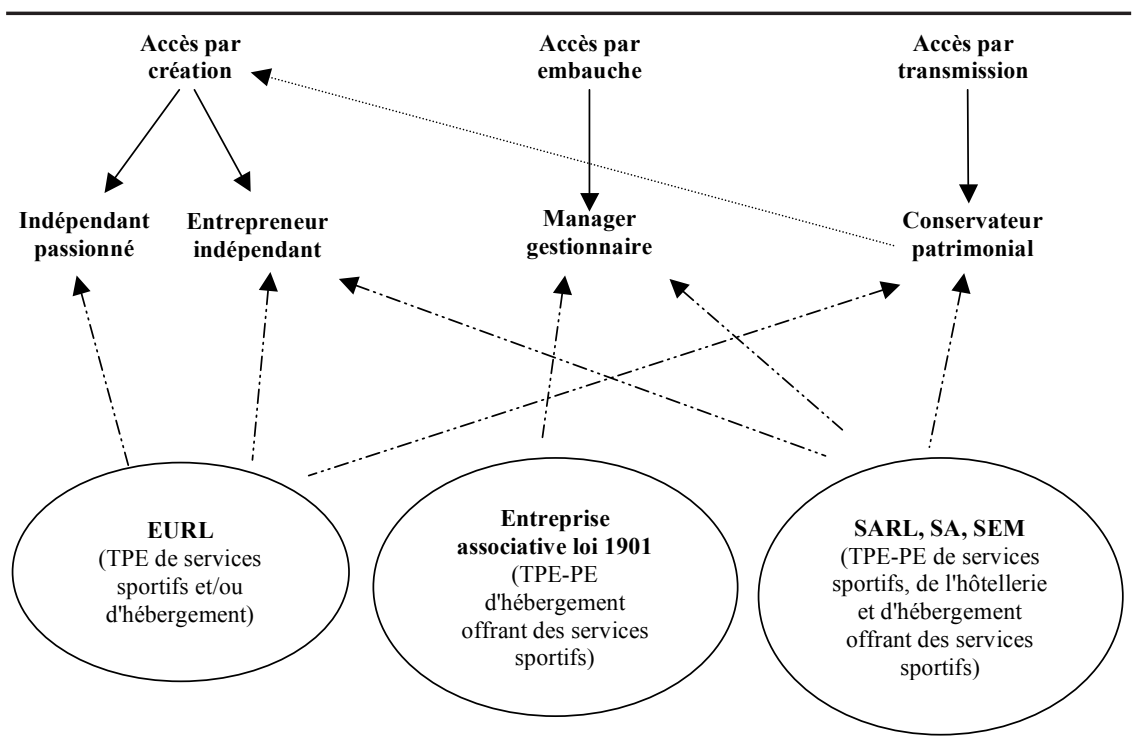

Chaque type de flèches simule une typologie fondée à partir d'un critère ou thème préalablement et théoriquement choisis. Par exemple, le mode d'accès n'aurait pas fait la différence entre l'IP et l'EI.

\section{Légende}

EURL: Entreprise unipersonnelle à responsabilité limitée.

SARL: Société anonyme à responsabilité limitée.

SA: Société anonyme.

SEM: Société d'économie mixte.

La simulation représentée par la figure 2 (ci-dessus), d'une part, confirme la variété et l'hétérogénéité des caractéristiques objectives des PE-TPE et, d'autre part, relativise l'effet de la taille et du statut juridique sur la structuration ou l'organisation de l'univers des PE-TPE. Si cela peut constituer un facteur de contingence de l'organisation de la grande entreprise industrielle (Mintzberg, 1982), il n'est pas vérifié pour la petite entreprise en général (Torrès, 1998) et du tourisme sportif en particulier.

De ce fait, l'application, dans une étude empirique, du concept de micromentalités semble réduire et homogénéiser la taxonomie en quatre types mettant en relation les objectifs et conceptions des dirigeants avec leurs comportements et choix économiques (formes organisationnelles, démarches stratégique et productive). En d'autres termes, la taille (effectif, chiffre d'affaires, parts de marché), le statut juridique, l'activité commercialisée, la 
démarche de production, etc., dans le cas de la petite entreprise, semblent refléter davantage une conjugaison des objectifs à long terme et projets sociaux des entrepreneurs dirigeants avec les contraintes de l'environnement et non un déterminisme à sens unique de ces dernières.

D'un autre côté, les résultats sur lesquels débouche ce modèle n'infirment pas les typologies classiques de l'entreprise artisanale (traditionnelle) et de l'entreprise managériale (moderne) auxquelles font référence plusieurs autres recherches. En effet, au regard de l'implication ou non de la famille, des produits offerts (sport ou hébergement), de la prise en compte ou non de la valeur patrimoniale de l'entreprise, etc., apparaissent parfois deux groupes d'entreprises avec deux tendances différentes: artisanale et managériale (Zarca, 1986). Mais apparaissent aussi des distinctions, au sein même de ces derniers, d'une part, au niveau de la signification de l'indépendance, des conceptions de l'entreprise et des rapports au marché et, d'autre part, au niveau des rapports au client, au marché, au produit, au niveau des formes organisationnelles, etc. Bref, ces distinctions confirment également la validité des modèles intermédiaires (Julien et Marchesnay, 1988).

Ces différences, étant davantage liées aux logiques d'action des dirigeants qui permettent d'orchestrer leurs objectifs parfois contradictoires eu égard à la rationalité du marché et dont l'impact est perceptible dans les formes organisationnelles des PE-TPE, conduisent alors à identifier les dimensions les plus structurantes de ces quatre types taxonomiques des PETPE et des dirigeants. Cela s'effectue, d'une part, à partir de la micromentalité du dirigeant identifiée à travers les trois dimensions suivantes: $a$ ) la signification et de la manière d'atteindre l'indépendance, $b$ ) la conception de l'entreprise et $c$ ) le rapport au marché, et, d'autre part, à partir des caractéristiques des PE-TPE: $d$ ) la forme d'organisation choisie pour l'entreprise, e) le mode de management et de production.

Le choix de ces dimensions ne signifie pas le rejet de celles prises en compte dans la construction des taxonomies ( $c f$. tableaux 1 à 4), mais les synthétisent et montrent leurs interrelations. En effet, par exemple l'indépendance, au premier degré (sans la prise en compte de sa signification relative pour le dirigeant), ne peut que réduire la typologie à un partage en deux groupes de dirigeants et d'entreprises, c'est-à-dire les entreprises des «nonsalariés » d'un côté et celles des «salariés », de l'autre (Gresle, 1981). Mais sa mise en relation avec les micromentalités, son importance et la manière de la réaliser permet de lui donner une dimension structurante synthétique.

En effet, pour le dirigeant IP, l'indépendance signifie le contraire du travail classique, c'est-à-dire l'absence de contraintes temporelles et spatiales dans l'activité professionnelle et donc la proximité de sa passion. Elle 
s'obtient par la résistance aux contraintes universelles du marché, en d'autres termes, par l'acquisition d'une liberté à l'égard des trois types de marché: des services, du travail et des capitaux.

Pour le dirigeant EI, c'est la liberté de création grâce au contrôle du capital de l'entreprise et du processus décisionnel. Pour le dirigeant CP, cela signifie l'isolement de l'entreprise de l'environnement extra-familial et l'évitement de son passage sous le contrôle (partiel ou intégral) d'un tiers étranger au cercle familial (méfiance à l'égard du marché du travail et des capitaux). Le MG, quant à lui, attache plus d'importance à l'indépendance financière de son entreprise et peu à sa propre indépendance d'où l'acceptation de sa condition de salarié.

Les micromentalités, à travers les différentes significations de l'indépendance, de l'entreprise (moyen, objet, outil et raison d'être) et des rapports au marché (résistance, instrumentalisation, coopération et soumission), induisent des logiques d'action différentes qui s'expriment par des modes de management, des orientations stratégiques et des démarches productives particulières.

Le présent travail a permis, à travers une approche compréhensive, de faire le lien entre le dirigeant et le mode de gestion de la petite entreprise. En effet, cette analyse statique a mis en avant l'efficacité des micromentalités des dirigeants sur leurs comportements et, par là même, a révélé le rôle «homogénéisateur» de ces dernières dans la construction d'une typologie taxonomique multicritère des PE-TPE et des entrepreneurs dirigeants.

Il a également permis de confirmer l'insuffisance des critères quantitatifs et objectifs liés à la petite entreprise et à son environnement économique pour construire des typologies réalistes et compréhensibles (Bentabet, Michun et Trouvé, 1999; Julien, 1997; Torrès, 1998). L’hétérogénéité de ces critères objectifs peut conduire soit à choisir des «typologies déductives » unidimensionnelles (Candau, 1981) fondées sur la taille, l'ancienneté, le statut juridique, etc., ou à multiplier les critères de classement et finir par produire des typologies «éclatées » ou casuistiques ne rendant pas compte de la réalité des PE-TPE (Bentabet, Michun et Trouvé, 1999).

La petite entreprise ne peut pas être réduite à une organisation formelle visant exclusivement l'efficience économique à tout prix. Ce qui conduit à accorder une importance aux micromentalités des entrepreneurs, à leurs projets socioprofessionnels pour comprendre la gestion de la très petite et petite entreprise.

Il n'est donc pas possible d'évaluer les performances de ces entreprises prioritairement et exclusivement à partir des critères économiques 
(rentabilité, dynamisme de l'investissement, etc.) ou marketing classiques (satisfaction du client, concurrence, etc.). Il est clair, bien que constituant des éléments de leurs actions, que les entrepreneurs dirigeants ne conçoivent pas la maximisation des profits et la satisfaction du client comme des objectifs majeurs (Bouhaouala et Chantelat, 2002). En somme, l'apparente «irrationalité» de certains comportements de dirigeants renverrait moins à un manque de compétences managériales et davantage à un ajustement de leurs comportements en lien avec leurs micromentalités.

\section{Bibliographie}

BAUER, M. (1993), Les patrons de PME entre le pouvoir, l'entreprise et la famille, Paris, InterÉditions.

Bentabet, E., S. Michun et P.Trouvé (1999), Gestion des hommes et formation dans les très petites entreprises, Centre d'études et de recherches sur les qualifications, étude $\mathrm{n}^{\circ} 72$.

Bouhaouala, M.(1999), Micro-mentalités et logiques d'action des dirigeants des petites entreprises du tourisme sportif, Thèse de doctorat, Université Joseph-Fourier, Grenoble I.

Bouhaouala, M. (2001), «Relations inter-entreprises dans un marché local: le cas des PE-TPE du tourisme sportif en Vercors», Revue Espaces et Sociétés, $\mathrm{n}^{\text {os }} 105-106$.

Bouhaouala, M. et P. Chantelat (2002), «Les pratiques commerciales des petites entreprises du tourisme sportif de nature: entre rationalité marketing et logiques sociales des dirigeants », Revue internationale PME, vol. 15, nº 1 .

Bouhaouala. M. et P. Chifflet (2001), «Logiques d'action des moniteurs des sports de pleine nature: entre passion et profession », Revue STAPS, ${ }^{\circ} 57$.

Candau, P. (1981), «Pour une taxonomie de l'hypofirme», Revue d'économie industrielle, $\mathrm{n}^{\circ} 16, \mathrm{p} .17-33$.

Coluins. M. et L. Randolph (1991), «Business or hobby? Small firms in sport and recreation», dans A.J. Veal et P. Jonson (dir.), Leisure and Tourism: Social and Economic Change, Sidney, University of Technology, p. 433-438.

Doty, D.H. et W.H. Glick (1994), «Typologies as a unique form of theory building: toward improved understanding and modeling», Academy of Management Review, vol. $19, \mathrm{n}^{\circ} 2$.

Gislain, J.J. et P. Steiner (1995), La sociologie économique 1890-1920, Paris, Presses universitaires de France.

GresLe, F. (1981), «L'indépendance professionnelle, actualité et portée de concept dans le cas français », Revue française de sociologie, XXII-4, p. 483-501.

Herscovici, A. (1994), Économie de la culture et de la communication, Paris, L'Harmattan. 
Joyal, A. (1986), «Les difficultés de travailler autrement au Québec», Loisir et Société, vol. $9, \mathrm{n}^{\circ}$ 2, p. 315-336.

Julien, P.-A. (1997), Les PME: bilan et perspectives, Québec, Presses InterUniversitaires.

Julien, P.-A. et M. MARChESNAY (1988), La petite entreprise: principes d'économie et de gestion, Paris, Vermette/Vuibert.

Julien. P.-A. et M. Marchesnay (1996), L'Entrepreneuriat, Paris, Economica.

LAhlou, S., J. MafFre et P. MoATi (1991), Régulation des marchés culturels : le rôle de la passion, Paris, CREDOC.

Mardellat, R. (1994), «Pratiques commerciales et représentations dans l'artisanat», dans J.C. Abric, Pratiques sociales et représentations, Paris, Presses universitaires de France, p. 145-177.

Miller, D. (1996), «Configurations revisited», Strategic Management Journal, vol. 17.

Mintzberg, H. (1982), Structure et dynamique des organisations, Paris, Les Éditions d'Organisation.

Montı, P. (1993), «Rationalité et marché des biens culturels», Problèmes économiques, $\mathrm{n}^{\circ} 2339$, p. 23-32.

Porter, M. (1982), Choix stratégiques et concurrence, réédition 1990, Paris, Économica.

Schumpeter, J. (1911), Théorie de l'évolution économique, Paris, Dalloz.

Simiand, F. (1932), Le salaire, l'évolution sociale et la monnaie, Paris, Alcan.

Simiand, F. (1934), «La monnaie, réalité sociale», Annales sociologiques, vol. 1.

Torrès, O. (1998), PME de nouvelles approches, Paris, Économica.

Weber. M. (1964), L'éthique protestante et l'esprit du capitalisme, $1^{\text {re }}$ édition 1905 , Paris, Plon.

ZARCA, B. (1986), L'artisanat français: du métier traditionnel au groupe social, Paris, Economica.

Revue internationale P.M.E., vol. 20, n 2, 2007

(c) 2007 - Presses de l'Université du Québec

Édifice Le Delta I, 2875, boul. Laurier, bureau 450, Québec, Québec G1V 2M2 • Tél.: (418) 657-4399 - www.puq.ca 